\title{
Evaluation of Cereals and Pseudocereals Suitability for the Development of New Probiotic Foods
}

\author{
Monika Kocková, Monika Dilongová, Eva Hybenová, and L’ubomír Valík \\ Department of Nutrition and Food Assessment, Institute of Biochemistry, Nutrition and Health Protection, \\ Faculty of Chemical and Food Technology, Slovak University of Technology in Bratislava, 81237 Bratislava, Slovakia
}

Correspondence should be addressed to Monika Kocková; monika.kockova@gmail.com

Received 6 May 2013; Revised 2 July 2013; Accepted 23 July 2013

Academic Editor: Yiannis Kourkoutas

Copyright (C) 2013 Monika Kocková et al. This is an open access article distributed under the Creative Commons Attribution License, which permits unrestricted use, distribution, and reproduction in any medium, provided the original work is properly cited.

\begin{abstract}
The aim of the present work was to evaluate suitability of cereals and pseudocereals for the development of new probiotic foods and to evaluate the stability of cereal and pseudocereal porridges fermented by probiotic strain Lactobacillus rhamnosus GG. Ten samples of cereals and pseudocereals obtained from Slovak mill house and markets were used in this work. A mixture of each cereal and pseudocereal samples with water $(10 \% \mathrm{w} / \mathrm{v})$ was inoculated after sterilization with coequal number of Lactobacillus rhamnosus GG, to obtain approximately 5-6 log colony form units per gram of suspensions. Fermentation was led at $37^{\circ} \mathrm{C}$ during 10 hours. Fermented suspensions were stored for 21 days at $5^{\circ} \mathrm{C}$. Monitoring of cell counts, $\mathrm{pH}$ value, and concentration of organic acids during fermentation and storage was done.
\end{abstract}

\section{Introduction}

Cereals and pseudocereals belong to the most important food for the majority of mankind. They are a good source of saccharides (especially starch and fibres), proteins (with good quality of amino acids, except lysine in case of cereal grains), lipids (essential fatty acids, almost no presence of saturated fatty acids), vitamins (B group), and minerals (calcium, potassium, magnesium, ferrum, zinc, cuprum, and phosphorus) $[1,2]$. On the other hand, antinutritive factors, such as phytic acid, tannins, polyphenols, and enzyme inhibitors, that decrease nutritional quality of grains by binding proteins, saccharides and mineral to complexes are naturally present in cereal and pseudocereal grains [3].

Cereals and pseudocereals are usually consumed in the form of bread, breakfast cereals, or cereal bars in developed countries. On the other hand, in developing countries the consumption of fermented cereals in the form of beverages, cakes, or porridges is common. These kinds of food would increase consumption of cereals and mainly pseudocereals in developed countries and moreover contribute to increase probiotic intake, in case of using probiotic strain for fermentation process [4].
Fermentation conduces to the enhancement of nutritional quality of end products via production of nutritive factors (e.g., biogenic amines and $\gamma$-aminobutyric acid) and reduction of antinutritive factors $[3,5]$. Fermentation also makes for improvement of sensory quality of end products in comparison to raw materials by producing aroma compounds by lactic acid bacteria $[3,6]$ and to the extension of shelf life, through the production of antimicrobial compounds $[7,8]$.

Probiotics are live microorganisms which have a positive effect on consumer [9]. Lactobacillus rhamnosus GG is one of the most monitored probiotic strains. It belongs to Grampositive, non-spore-forming, nonmotile, catalase-negative, facultatively anaerobic or microaerophilic, and mesophilic bacteria [10]. Metabolism of Lb. rhamnosus GG is facultatively heterofermentative; it can produce acetic acid, formic acid and ethanol, in addition to lactic acid, in lack of glucose in fermentation environment [11]. Lb. rhamnosus GG is highly tolerant to acidic environment of the stomach; it is able to survive in intestinal passage, adhere to intestinal mucosa, and colonize gastrointestinal tract after three days of consumption $[12,13]$. It enhances human natural resistance and healthy digestive system and inhibits adhesion of some 
pathogenic bacteria. It relieves syndromes of irritation of GI tract, atopic dermatitis, and cow milk allergy $[14,15]$.

In Slovak food market, probiotic foods are mainly milk based, and therefore probiotics are not available for all consumers, especially for those who suffer from milk allergies and intolerances. This was the reason why we focused our research on the selection of plant materials (cereals, pseudocereals, and legumes) on the bases of growth and metabolic characteristics of selected probiotic strain, Lactobacillus rhamnosus GG, which will lead to the development of new probiotic foods.

\section{Materials and Methods}

2.1. Starter Culture. The probiotic strain of Lb. rhamnosus GG was used in this work. It was provided by Dr. Salminen (University of Turku, Turku, Finland) through mediation of Dr. Lauková (State Veterinary and Food Institute, Košice, Slovakia).

2.2. Cereal and Pseudocereal Substrates. Ten samples of cereals and pseudocereals were used in this work. Rye flour (RF), rye grain $(\mathrm{RG})$, barley flour $(\mathrm{BF})$, and whole barley flour (WBF), amaranth flour (AF), buckwheat flour (BWF), whole buckwheat flour (WBWF), whole oat flour (WOF) were obtained from mill house (Mlyn Zrno, Šíšov, Slovak Republic); amaranth grain (AG, Primeal, Peaugres, France) and millet grain (MG, Marianna wholesale, Ivánka pri Dunaji, Slovak Republic) were obtained from market. Sixty grams of flours or milled and sieved grains were mixed with $540 \mathrm{~mL}$ of deionised water for preparation of fermentation substrates.

2.3. Fermentation and Storage. Substrates were inoculated after autoclaving for $15 \mathrm{~min}$ at $121^{\circ} \mathrm{C}$ and cooling down with overnight culture (MRS broth, 18 hours, $37^{\circ} \mathrm{C}$ ) of $L b$. rhamnosus GG to give approximately 5 to $6 \log$ colony form units per gram of suspensions. Static fermentation was performed for 10 hours (according to the previous results) at $37^{\circ} \mathrm{C}$. Samples for analyses were taken every 2 hours. Storage observations were carried out at $5^{\circ} \mathrm{C}$ for 21 days. Samples for analyses were taken every 2-3 days.

2.4. Viable Cell Enumeration. Enumeration of viable cells of Lb. rhamnosus GG was performed by estimation of colony forming unit number on MRS-agar plates according to the STN ISO 15214.

2.5. Determination of $\mathrm{pH}$ and Organic Acids. The $\mathrm{pH}$ of samples was measured by pH-meter CG 843 (Schott, Mainz, Germany). The quality and quantity of the produced organic acids were measured by isotachophoretic analysis by using the Isotachophoretic Analyser ZKI 01 (Villa Labeco, Spišská Nová Ves, Slovak Republic). Electrolytic system according to Kocková et al. [16] was used. Quantitative analysis was performed by calibration of standard solution of lactic, acetic, citric, formic, and succinic acids (Lachema, Brno, Czech Republic).
2.6. Estimation of Growth and Metabolic Parameters of Lb. rhamnosus GG. Growth curves of Lb. rhamnosus GG in each substrate were modelled with a mechanistic model of Baranyi and Roberts [17]. Growth and metabolic parameters were calculated from each curve.

2.7. Statistical Analyses. Each experiment was performed in three separate trials. Results represented means with standard deviations. Statistical analyses were carried out using Microsoft Excel 2007 (Microsoft, Redmond, WA, USA). Data were treated by ANOVA test with a least significant difference of $95 \%$.

\section{Results and Discussion}

3.1. Fermentation. Fermentation of cereal and pseudocereal substrates for evaluation of their suitability for lactic acid fermentation was carried out. Cell counts, $\mathrm{pH}$ value, and organic acid concentrations were monitored in fixed period. Growth and metabolic curves are shown in Figure 1 and growth and metabolic parameters in Tables 1-5.

As it is shown in Figure 1, probiotic strain Lb. rhamnosus GG was able to grow and metabolize in each cereal and pseudocereal substrate during a ten-hour fermentation process. Lb. rhamnosus GG was able to grow from initial cell counts 5.04-6.46 $\log \mathrm{cfu} \mathrm{g}^{-1}$ to final 7.40-8.80 $\log \mathrm{cfu} \mathrm{g}^{-1}$ (Table 1), which is similar to density of $L b$. rhamnosus GG reached in milk [10], in cereal water-based puddings [18], and maize porridges with barley addition [19]. The highest growth rate was calculated in case of amaranth flour (in which the longest lag phase was observed), $0.589 \log \mathrm{cfu} \mathrm{g}^{-1} \mathrm{~h}^{-1}$. The lowest growth rate was calculated for whole oat flour, $0.248 \log \mathrm{cfu} \mathrm{g}^{-1} \mathrm{~h}^{-1}$ (Table 1). Growth rate of Lb. rhamnosus GG during fermentation of milk at $35^{\circ} \mathrm{C}$ was $0.653 \log \mathrm{cfu} \mathrm{mL} \mathrm{m}^{-1} \mathrm{~h}^{-1}[10]$. Higher growth rate was found during fermentation of amaranth milk- and water-based puddings and buckwheat milk-based pudding by $L b$. rhamnosus GG [20]. Time to double ranged from $0.51 \mathrm{~h}$ (amaranth flour) to $1.21 \mathrm{~h}$ (whole oat flour). Lag phase, time for adapting microorganisms to new environment, ranged from $0.73 \mathrm{~h}$ (rye grain) to $3.88 \mathrm{~h}$ (amaranth flour). In substrate from millet grain, no lag phase was observed (Table 1).

During fermentation, organic acids production, as a result of metabolic activity of $L b$. rhamnosus GG, was observed. This caused decreasing of $\mathrm{pH}$ values in each substrate. $\mathrm{pH}$ values dropped from initial 4.93-6.08 to final 4.315.99 (Figure 1, Table 2). Final pH value caused by metabolic activity of $L b$. rhamnosus GG in MRS broth was 4.00 [21], in milk 6.50 [10] and in milk-based cereal puddings and maize porridges with barley under 4.00 [18, 19]. In case of substrates from amaranth grain, buckwheat flour, and millet grain, a long lag phase of reducing $\mathrm{pH}$ values (7.90, 8.70 , and $6.50 \mathrm{~h}$, resp.) was observed. In these substrates, higher rate of reducing $\mathrm{pH}\left(-0.355,-1.063\right.$, and $-0.244 \mathrm{~h}^{-1}$, resp.) was calculated. Rates of decreasing $\mathrm{pH}$ values in other substrates ranged from $-0.003 \mathrm{~h}^{-1}$ (whole buckwheat flour) to $-0.047 \mathrm{~h}^{-1}$ (barley flour) (Table 2). In amaranth 


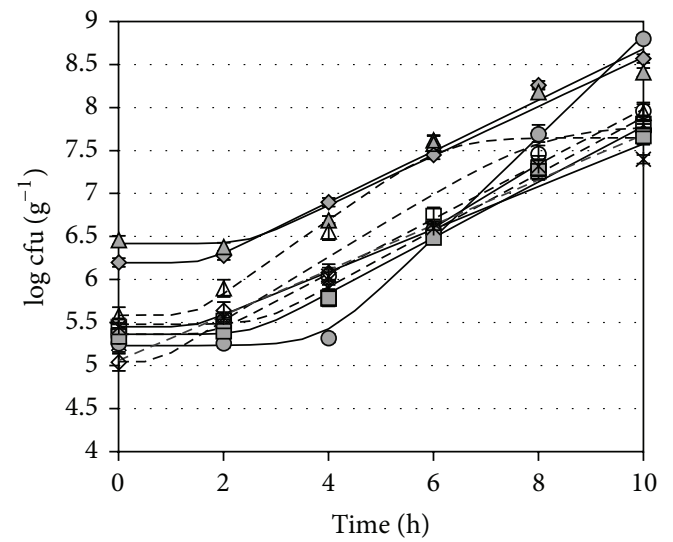

(a)

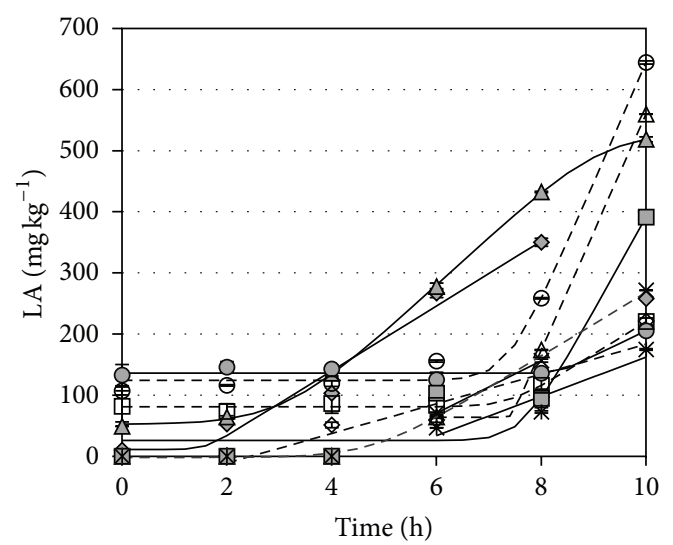

(c)

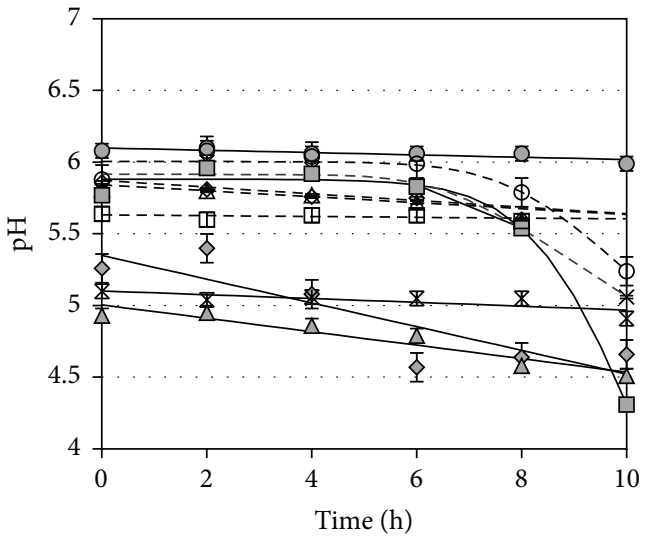

(b)

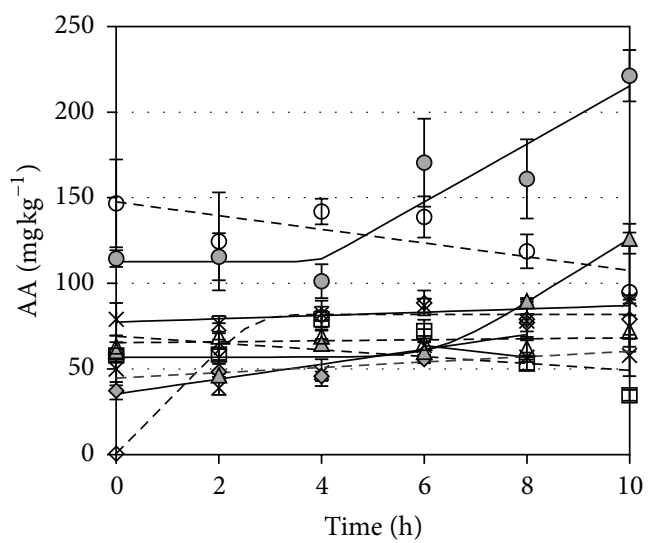

(d)

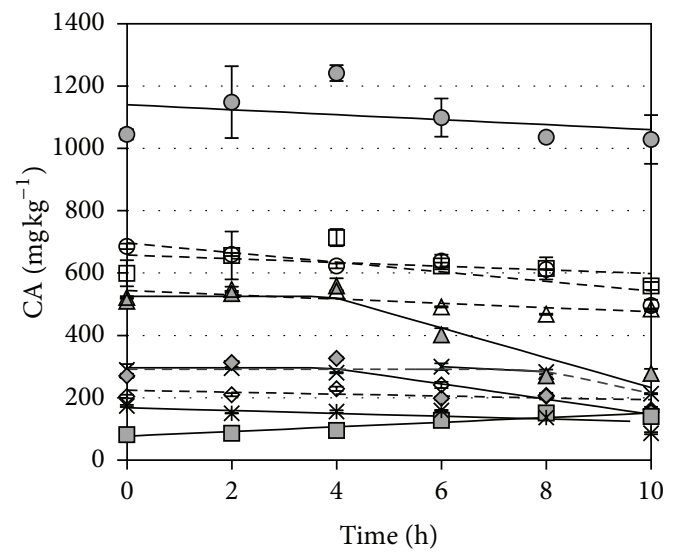

\footnotetext{
$\diamond$ Rye flour (RF)

$-\diamond-$ Rye grain (RG)

$\neg-$ Barley flour (BF)

-曰- Whole buckwheat flour (WBWF)

$-A$ - Whole barley flour (WBF)

* Whole oat flour (WOF)

$-\rtimes$ - Millet grain (MG)

-O- Amaranth flour (AF)

CFU: colony form units

- $\Theta$ - Amaranth grain (AG)

LA: lactic acid

AA: acetic acid

$\neg-$ Buckwheat flour (BWF)

CA: citric acid
}

(e)

FIGURE 1: Evaluation of cell counts of Lb. rhamnosus GG (a) and changes in pH values (b) and concentration of lactic acid (c), acetic acid (d), and citric acid (e) during fermentation. 
TABLE 1: Growth parameters of $L b$. rhamnosus GG during fermentation of cereal and pseudocereal substrates.

\begin{tabular}{lccccc}
\hline Substrate & Gr $\left[\log c f u g^{-1} h^{-1}\right]$ & $t_{d}[\mathrm{~h}]$ & $\lambda[\mathrm{h}]$ & $N_{0}\left[\log c f u g^{-1}\right]$ & $N_{\max }\left[\log c f u g^{-1}\right]$ \\
\hline RF & $0.297^{\mathrm{b}}$ & $1.01^{\mathrm{d}}$ & $1.64^{\mathrm{c}}$ & $6.20^{\mathrm{f}}$ & $8.57^{\mathrm{f}}$ \\
RG & $0.372^{\mathrm{c}}$ & $0.81^{\mathrm{c}}$ & $0.73^{\mathrm{a}}$ & $5.05^{\mathrm{a}}$ & $7.78^{\mathrm{c}}$ \\
BF & $0.287^{\mathrm{b}}$ & $1.05^{\mathrm{d}}$ & $2.44^{\mathrm{d}}$ & $6.42^{\mathrm{g}}$ & $8.41^{\mathrm{e}}$ \\
WBF & $0.430^{\mathrm{d}}$ & $0.70^{\mathrm{b}}$ & $1.42^{\mathrm{b}}$ & $5.59^{\mathrm{e}}$ & $7.65^{\mathrm{b}}$ \\
AF & $0.589^{\mathrm{e}}$ & $0.51^{\mathrm{a}}$ & $3.88^{\mathrm{e}}$ & $5.23^{\mathrm{b}}$ & $8.80^{\mathrm{g}}$ \\
AG & $0.319^{\mathrm{b}}$ & $0.94^{\mathrm{c}}$ & $1.79^{\mathrm{c}}$ & $5.36^{\mathrm{c}}$ & $7.96^{\mathrm{d}}$ \\
BWF & $0.324^{\mathrm{b}}$ & $0.93^{\mathrm{c}}$ & $2.53^{\mathrm{d}}$ & $5.36^{\mathrm{c}}$ & $7.67^{\mathrm{b}}$ \\
WBWF & $0.328^{\mathrm{b}}$ & $0.92^{\mathrm{c}}$ & $2.68^{\mathrm{d}}$ & $5.48^{\mathrm{d}}$ & $7.81^{\mathrm{c}}$ \\
WOF & $0.248^{\mathrm{a}}$ & $1.21^{\mathrm{d}}$ & $1.42^{\mathrm{b}}$ & $5.45^{\mathrm{d}}$ & $7.40^{\mathrm{a}}$ \\
MG & $0.263^{\mathrm{a}}$ & $1.15^{\mathrm{d}}$ & - & $5.06^{\mathrm{a}}$ & $7.67^{\mathrm{b}}$ \\
\hline
\end{tabular}

RF: rye flour, RG: rye grain, BF: barley flour, WBF: whole barley flour, AF: amaranth flour, AG: amaranth grain, BWF: buckwheat flour, WBWF: whole buckwheat flour, WOF: whole oat flour, MG: millet grain.

Gr: growth rate, $t_{d}$ : time to double, $\lambda$ : lag phase, $N_{0}$ : initial density of $L b$. rhamnosus $\mathrm{GG}, N_{\max }$ : final density of $L b$. rhamnosus GG.

${ }^{\mathrm{a}-\mathrm{g}}$ Means within a column with different superscript letters are significantly different $(P<0.05)$.

TABLE 2: Parameters of $\mathrm{pH}$ value changes during fermentation of cereal and pseudocereal substrates with Lb. rhamnosus GG.

\begin{tabular}{lcccc}
\hline Substrate & $k_{\mathrm{pH}}\left[\mathrm{h}^{-1}\right]$ & $\lambda_{\mathrm{pH}}[\mathrm{h}]$ & $\mathrm{pH}_{0}$ & $\mathrm{pH}_{\text {end }}$ \\
\hline RF & $-0.083^{\mathrm{f}}$ & - & $5.35^{\mathrm{b}}$ & $4.62^{\mathrm{b}}$ \\
RG & $-0.029^{\mathrm{d}}$ & - & $5.87^{\mathrm{d}}$ & $5.60^{\mathrm{e}}$ \\
BF & $-0.047^{\mathrm{e}}$ & - & $5.00^{\mathrm{a}}$ & $4.78^{\mathrm{b}}$ \\
WBF & $-0.026^{\mathrm{d}}$ & - & $5.84^{\mathrm{d}}$ & $5.59^{\mathrm{e}}$ \\
AF & $-0.008^{\mathrm{b}}$ & - & $6.10^{\mathrm{e}}$ & $5.99^{\mathrm{f}}$ \\
AG & $-0.355^{\mathrm{g}}$ & $7.90^{\mathrm{b}}$ & $6.00^{\mathrm{e}}$ & $5.24^{\mathrm{d}}$ \\
BWF & $-1.063^{\mathrm{h}}$ & $8.70^{\mathrm{c}}$ & $5.88^{\mathrm{d}}$ & $4.31^{\mathrm{a}}$ \\
WBWF & $-0.003^{\mathrm{a}}$ & - & $5.63^{\mathrm{c}}$ & $5.59^{\mathrm{e}}$ \\
WOF & $-0.013^{\mathrm{c}}$ & - & $5.10^{\mathrm{a}}$ & $4.91^{\mathrm{c}}$ \\
MG & $-0.244^{\mathrm{g}}$ & $6.50^{\mathrm{a}}$ & $5.91^{\mathrm{d}}$ & $5.06^{\mathrm{c}}$ \\
\hline
\end{tabular}

RF: rye flour, RG: rye grain, BF: barley flour, WBF: whole barley flour, AF: amaranth flour, AG: amaranth grain, BWF: buckwheat flour, WBWF: whole buckwheat flour, WOF: whole oat flour, MG: millet grain.

$k_{\mathrm{pH}}$ : rate of changes in $\mathrm{pH}$ values, $\lambda_{\mathrm{pH}}$ : lag phase of $\mathrm{pH}$ changes, $\mathrm{pH}_{0}$ : initial $\mathrm{pH}, \mathrm{pH}_{\text {end }}$ : final $\mathrm{pH}$.

${ }^{\mathrm{a}-\mathrm{h}}$ Means within a column with different superscript letters are significantly different $(P<0.05)$.

and buckwheat water- and milk-based pudding rates of $\mathrm{pH}$ value decreasing ranged from 0.144 to $0.317 \mathrm{~h}^{-1}$ [20].

The concentration of lactic acid at the beginning of fermentation process was established from $11.27 \mathrm{mg} \mathrm{kg}^{-1}$ in case of rye flour to $136.40 \mathrm{mg} \mathrm{kg}^{-1}$ in case of amaranth flour. In substrates from rye grain, buckwheat flour, whole oat flour, and millet grain concentration of lactic acid was under detection limit. The increase in lactic acid concentration was observed in all substrates (Figure 1, Table 3). The highest rate of production of lactic acid was calculated for whole barley flour and amaranth grain, 192.85 and $195.91 \mathrm{mg} \mathrm{kg}^{-1} \mathrm{~h}^{-1}$, respectively. In these substrates, long lag phase of lactic acid production was observed, 7.43 and $7.35 \mathrm{~h}$, respectively. Long lag phase was observed also in case of amaranth flour, buckwheat flour and whole buckwheat flour, ranged
TABLE 3: Parameters of lactic acid concentration changes during fermentation of cereal and pseudocereal substrates with Lb. rhamnosus GG.

\begin{tabular}{lcccc}
\hline Substrate & $\begin{array}{c}k_{\text {acid }} \\
{\left[\mathrm{mg} \mathrm{kg}^{-1} \mathrm{~h}^{-1}\right]}\end{array}$ & $\lambda_{\text {acid }}[\mathrm{h}]$ & $c_{0}\left[\mathrm{mg} \mathrm{kg}^{-1}\right]$ & $c_{\text {end }}\left[\mathrm{mg} \mathrm{kg}^{-1}\right]$ \\
\hline RF & $53.07^{\mathrm{d}}$ & $1.58^{\mathrm{a}}$ & $11.27^{\mathrm{b}}$ & $349.98^{\mathrm{d}}$ \\
RG & $24.31^{\mathrm{a}}$ & $2.50^{\mathrm{b}}$ & $0.00^{\mathrm{a}}$ & $221.16^{\mathrm{b}}$ \\
BF & $78.81^{\mathrm{e}}$ & $3.12^{\mathrm{c}}$ & $52.51^{\mathrm{c}}$ & $529.31^{\mathrm{f}}$ \\
WBF & $192.85^{\mathrm{g}}$ & $7.43^{\mathrm{e}}$ & $64.14^{\mathrm{d}}$ & $559.60^{\mathrm{g}}$ \\
AF & $40.42^{\mathrm{c}}$ & $8.30^{\mathrm{g}}$ & $136.40^{\mathrm{g}}$ & $205.15^{\mathrm{b}}$ \\
AG & $195.91^{\mathrm{g}}$ & $7.35^{\mathrm{e}}$ & $124.09^{\mathrm{f}}$ & $644.21^{\mathrm{h}}$ \\
BWF & $152.95^{\mathrm{f}}$ & $7.61^{\mathrm{f}}$ & $0.00^{\mathrm{a}}$ & $391.14^{\mathrm{e}}$ \\
WBWF & $53.22^{\mathrm{d}}$ & $7.38^{\mathrm{e}}$ & $81.05^{\mathrm{e}}$ & $220.40^{\mathrm{b}}$ \\
WOF & $32.02^{\mathrm{b}}$ & $5.00^{\mathrm{d}}$ & $0.00^{\mathrm{a}}$ & $174.66^{\mathrm{a}}$ \\
MG & $51.83^{\mathrm{d}}$ & $4.80^{\mathrm{d}}$ & $0.00^{\mathrm{a}}$ & $271.47^{\mathrm{c}}$ \\
\hline
\end{tabular}

RF: rye flour, RG: rye grain, BF: barley flour, WBF: whole barley flour, AF: amaranth flour, AG: amaranth grain, BWF: buckwheat flour, WBWF: whole buckwheat flour, WOF: whole oat flour, MG: millet grain.

$k_{\text {acid }}$ : rate of lactic acid concentration changes, $\lambda_{\text {acid }}$ lag phase of lactic acid concentration changes, $c_{0}$ : initial concentration of lactic acid, $c_{\text {end }}$ : final concentration of lactic acid.

${ }^{\mathrm{a}-\mathrm{h}}$ Means within a column with different superscript letters are significantly different $(P<0.05)$.

from 7.38 to $8.30 \mathrm{~h}$. The slowest lactic acid production was calculated in substrate from rye grain, $24.31 \mathrm{mg} \mathrm{kg}^{-1} \mathrm{~h}^{-1}$. Lactic acid level at the end of fermentation process ranged from $174.66 \mathrm{mg} \mathrm{kg}^{-1}$ (whole oat flour) to $644.21 \mathrm{mg} \mathrm{kg}^{-1}$ (amaranth grain) (Table 3). Lactic acid concentration after 18 hours fermentation by LAB in MRS broth was $36 \mathrm{~g} \mathrm{~L}^{-1}$ and in milk lower than $900 \mathrm{mg} \mathrm{L}^{-1}$ [21]. In maize porridges fermented by $L b$. rhamnosus GG, lactic acid concentrations were $3500 \mathrm{mg} \mathrm{kg}^{-1}$ and $4000 \mathrm{mg} \mathrm{kg}^{-1}$, respectively [19]. Lactic acid level in cereal water-based pudding fermented by Lb. rhamnosus GG was $2600 \mathrm{mg} \mathrm{kg}^{-1}$ and in milk-based 
TABLE 4: Parameters of acetic acid concentration changes during fermentation of cereal and pseudocereal substrates with $L b$. rhamnosus GG.

\begin{tabular}{lcccc}
\hline Substrate & $\begin{array}{c}k_{\text {acid }} \\
{\left[\mathrm{mg} \mathrm{kg}^{-1} \mathrm{~h}^{-1}\right]}\end{array}$ & $\lambda_{\text {acid }}[\mathrm{h}]$ & $c_{0}\left[\mathrm{mg} \mathrm{kg}^{-1}\right]$ & $c_{\text {end }}\left[\mathrm{mg} \mathrm{kg}^{-1}\right]$ \\
\hline RF & $4.34^{\mathrm{f}}$ & - & $35.44^{\mathrm{b}}$ & $76.98^{\mathrm{c}}$ \\
RG & $30.59^{\mathrm{h}}$ & - & $0.50^{\mathrm{a}}$ & $79.01^{\mathrm{c}}$ \\
BF & $18.64^{\mathrm{g}}$ & $6.28^{\mathrm{b}}$ & $56.93^{\mathrm{d}}$ & $126.06^{\mathrm{e}}$ \\
WBF & $0.29^{\mathrm{c}}$ & - & $65.29^{\mathrm{e}}$ & $72.14^{\mathrm{c}}$ \\
AF & $16.92^{\mathrm{g}}$ & $3.95^{\mathrm{a}}$ & $112.77^{\mathrm{g}}$ & $221.21^{\mathrm{f}}$ \\
AG & $-3.99^{\mathrm{a}}$ & - & $147.53^{\mathrm{h}}$ & $94.87^{\mathrm{d}}$ \\
BWF & - & - & - & - \\
WBWF & $-1.99^{\mathrm{b}}$ & - & $69.12^{\mathrm{e}}$ & $34.61^{\mathrm{a}}$ \\
WOF & $0.97^{\mathrm{d}}$ & - & $77.35^{\mathrm{f}}$ & $90.64^{\mathrm{d}}$ \\
MG & $1.56^{\mathrm{e}}$ & - & $44.68^{\mathrm{c}}$ & $57.87^{\mathrm{b}}$ \\
\hline
\end{tabular}

RF: rye flour, RG: rye grain, BF: barley flour, WBF: whole barley flour, AF: amaranth flour, AG: amaranth grain, BWF: buckwheat flour, WBWF: whole buckwheat flour, WOF: whole oat flour, MG: millet grain.

$k_{\text {acid }}$ : rate of acetic acid concentration changes, $\lambda_{\text {acid }}$ lag phase of acetic acid concentration changes, $c_{0}$ : initial concentration of acetic acid, $c_{\text {end }}$ : final concentration of acetic acid.

${ }^{\mathrm{a}-\mathrm{h}}$ Means within a column with different superscript letters are significantly different $(P<0.05)$.

TABLE 5: Parameters of citric acid concentration changes during fermentation of cereal and pseudocereal substrates with Lb. rhamnosus GG.

\begin{tabular}{lcccc}
\hline Substrate & $\begin{array}{c}k_{\text {acid }} \\
{\left[\mathrm{mg} \mathrm{kg}^{-1} \mathrm{~h}^{-1}\right]}\end{array}$ & $\lambda_{\text {acid }}[\mathrm{h}]$ & $c_{0}\left[\mathrm{mg} \mathrm{kg}^{-1}\right]$ & $c_{\text {end }}\left[\mathrm{mg} \mathrm{kg}^{-1}\right]$ \\
\hline RF & $-24.36^{\mathrm{g}}$ & $3.90^{\mathrm{a}}$ & $295.49^{\mathrm{d}}$ & $153.70^{\mathrm{c}}$ \\
RG & $-3.06^{\mathrm{b}}$ & - & $223.86^{\mathrm{c}}$ & $161.56^{\mathrm{c}}$ \\
BF & $-47.79^{\mathrm{i}}$ & $3.88^{\mathrm{a}}$ & $525.03^{\mathrm{e}}$ & $278.35^{\mathrm{e}}$ \\
WBF & $-6.80^{\mathrm{e}}$ & - & $543.68^{\mathrm{f}}$ & $484.96^{\mathrm{f}}$ \\
AF & $-8.02^{\mathrm{e}}$ & - & $1140.13^{\mathrm{h}}$ & $1028.97^{\mathrm{h}}$ \\
AG & $-15.40^{\mathrm{f}}$ & - & $696.34^{\mathrm{g}}$ & $496.19^{\mathrm{f}}$ \\
BWF & $7.43^{\mathrm{a}}$ & - & $76.71^{\mathrm{a}}$ & $140.23^{\mathrm{b}}$ \\
WBWF & $-5.97^{\mathrm{d}}$ & - & $657.78^{\mathrm{g}}$ & $559.08^{\mathrm{g}}$ \\
WOF & $-4.49^{\mathrm{c}}$ & - & $167.64^{\mathrm{b}}$ & $68.36^{\mathrm{a}}$ \\
MG & $-37.40^{\mathrm{h}}$ & $7.91^{\mathrm{b}}$ & $291.26^{\mathrm{d}}$ & $213.22^{\mathrm{d}}$ \\
\hline
\end{tabular}

RF: rye flour, RG: rye grain, BF: barley flour, WBF: whole barley flour, AF: amaranth flour, AG: amaranth grain, BWF: buckwheat flour, WBWF: whole buckwheat flour, WOF: whole oat flour, MG: millet grain.

$k_{\text {acid }}$ : rate of citric acid concentration changes, $\lambda_{\text {acid }}$ lag phase of citric acid concentration changes, $c_{0}$ : initial concentration of citric acid, $c_{\text {end }}$ f final concentration of citric acid.

${ }^{\mathrm{a}-\mathrm{i}}$ Means within a column with different superscript letters are significantly different $(P<0.05)$.

$9800 \mathrm{mg} \mathrm{kg}^{-1}$ [18]. Rates of production of lactic acid in buckwheat and amaranth water-based puddings during 8 hours fermentation were $162 \mathrm{mg} \mathrm{L}^{-1} \mathrm{~h}^{-1}$ and $280 \mathrm{mg} \mathrm{L}^{-1} \mathrm{~h}^{-1}$, respectively [20].

The rate of acetic acid production was slower compared to rate of lactic acid production, ranged from $0.29 \mathrm{mg} \mathrm{kg}^{-1} \mathrm{~h}^{-1}$ (whole barley flour) to $30.59 \mathrm{mg} \mathrm{kg}^{-1} \mathrm{~h}^{-1}$ (rye grain), which was good for final sensory quality of substrates. In case of amaranth grain and whole buckwheat flour, reduction in acetic acid level was observed and in substrate from buckwheat flour concentration of acetic acid was under detection limit during process (Figure 1, Table 4). Generally, production of acetic acid in our substrates was lower in compare to production of acetic acid in MRS broth or milk by the same probiotic strain [21].

Reducing in citric acid level was observed in all substrates, except those prepared from buckwheat flour. In case of rye flour, barley flour and millet grain, lag phase of reducing citric acid was observed and calculated, what caused higher rate of reducing citric acid, ranged from -24.36 to $-47.79 \mathrm{mg} \mathrm{kg}^{-1} \mathrm{~h}^{-1}$. In other substrates, rate of reducing citric acid ranged from -3.06 (rye grain) to $-15.40 \mathrm{mg} \mathrm{kg}^{-1} \mathrm{~h}^{-1}$ (amaranth grain) (Figure 1, Table 5). Partial utilization of citric acid by $L b$. rhamnosus GG was observed in maize porridges [19] and also in cereal water- and milk-based puddings [18].

Only small insignificant $(P>0.05)$ changes in formic and succinic acids content during fermentation were observed in all substrates (results are no shown), which concurs with the results of Helland et al. [19].

3.2. Storage. During storage at refrigerated temperature, changes in cell counts and metabolic activity were evaluated. For probiotic food, it is necessary to contain probiotic strain at certain level (6 log cfu per gram or millilitre of products, at least), and any metabolic activity of present microorganism is important.

As shown in Figure 2, density of $L b$. rhamnosus GG after 21 days of storage was over the limit required for probiotic food, except substrate prepared from whole buckwheat flour, in which cell counts of $L b$. rhamnosus GG decreased with growth rate $-0.00517 \log \mathrm{cfu} \mathrm{g}^{-1} \mathrm{~h}^{-1}$. Visible reduction in density of $L b$. rhamnosus GG was observed also in case of rye, barley, and amaranth flours (Figure 2, Table 6).

All fermented porridges were metabolically stabile except those prepared from amaranth flour, in which production of lactic acid $\left(3.752 \mathrm{mg} \mathrm{kg}^{-1} \mathrm{~h}^{-1}\right)$ and reduction of citric acid $\left(-0.732 \mathrm{mg} \mathrm{kg}^{-1} \mathrm{~h}^{-1}\right)$ and $\mathrm{pH}$ values $\left(-0.00435 \mathrm{~h}^{-1}\right)$ continued, and from whole buckwheat flour, in which metabolic activity continued during first five days of the storage period (Figure 2, Tables 7, 8, 9, and 10). According to these results, we evaluated used substrates as metabolically stabile, except substrate prepared from amaranth flour, in which higher content of saccharides, proteins, and lipids were found [16]. Evaluation of stability and definition of shelf-life of fermented cereal products was done only in scarce research. Angelov et al. [22] estimated shelf-life of probiotic oat drink to 21 days as a result of 24-day observation during storage period.

\section{Conclusion}

According to our results, we can say that probiotic strain $L b$. rhamnosus GG is able to grow and metabolize during the fermentation of cereal and pseudocereal substrates dealing to 


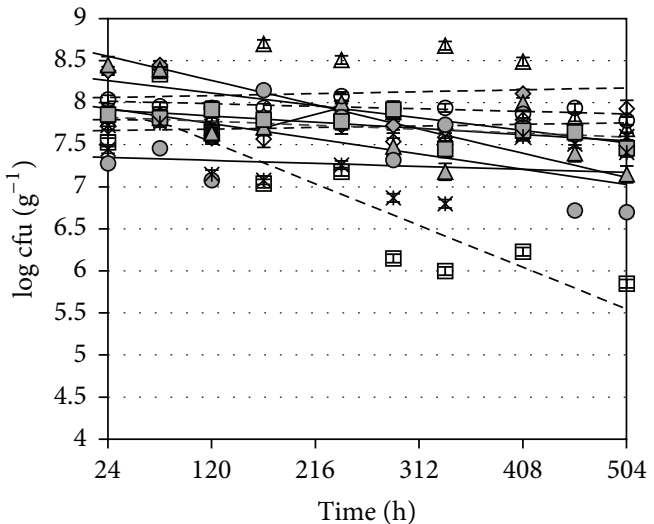

(a)

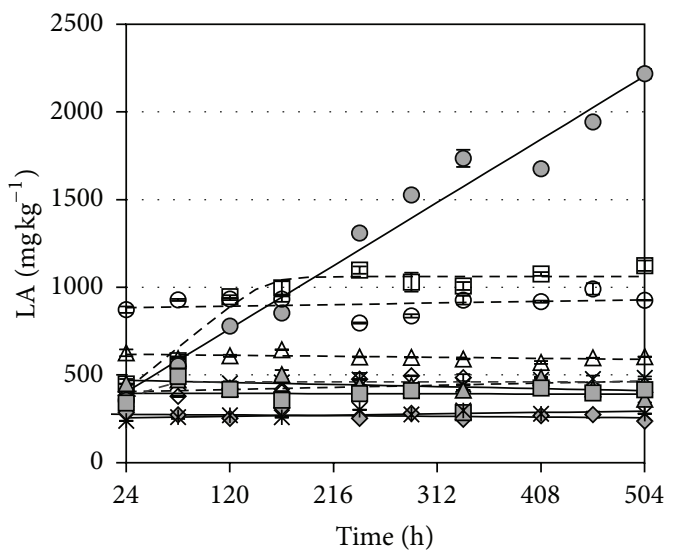

(c)

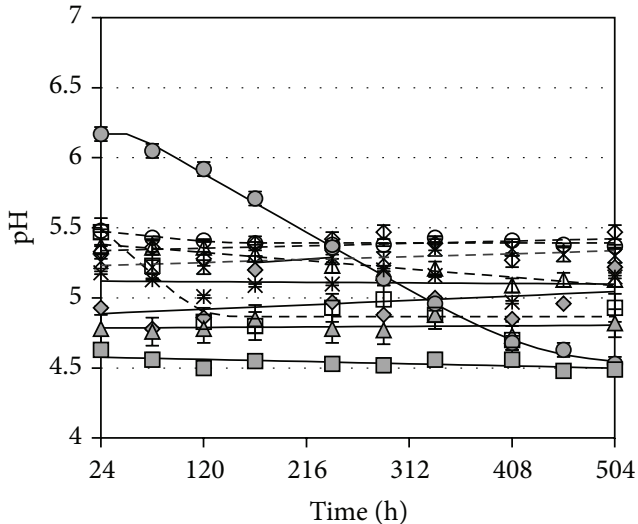

(b)

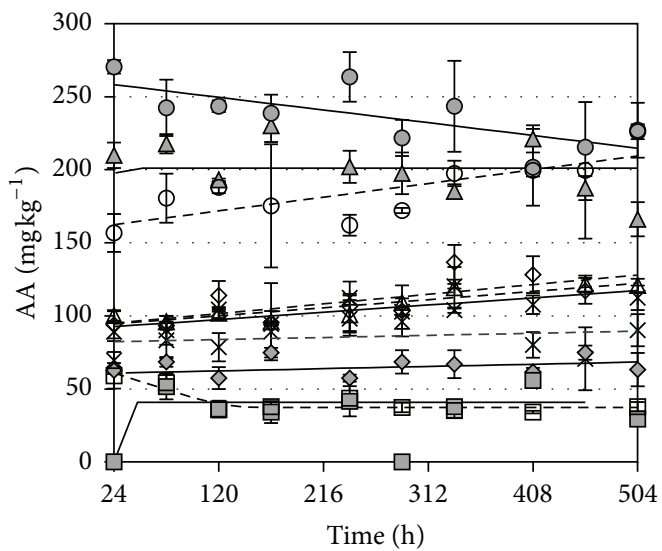

(d)

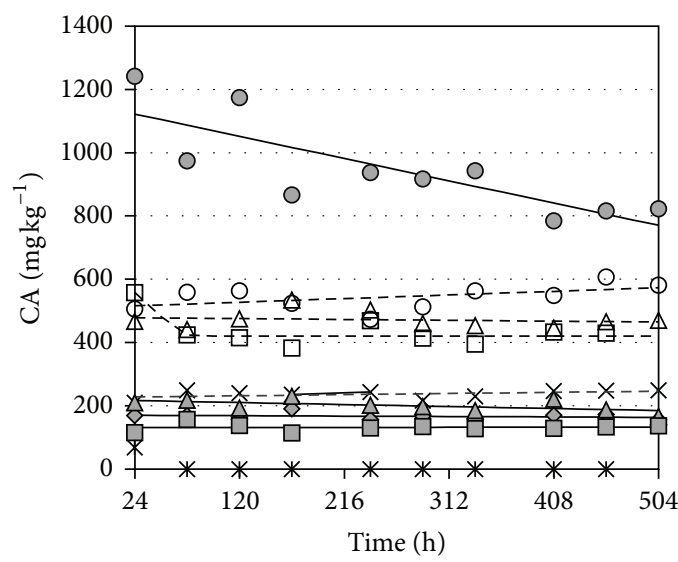

$\checkmark$ Rye flour (RF)

$-\diamond$ - Rye grain (RG)

$\checkmark-$ Barley flour (BF)

$-A$ - Whole barley flour (WBF)

-O- Amaranth flour (AF)

$-\Theta-$ Amaranth grain (AG)

$\neg-$ Buckwheat flour (BWF)
- - Whole buckwheat flour (WBWF)

* Whole oat flour (WOF)

$-\star$ - Millet grain (MG)

CFU: colony form units

LA: lactic acid

AA: acetic acid

CA: citric acid

(e)

FIGURE 2: Evaluation of cell counts of Lb. rhamnosus GG (a) and changes in pH values (b), and concentration of lactic acid (c), acetic acid (d) and citric acid (e) during storage of fermented cereal and pseudocereal substrates. 
TABLE 6: Growth parameters of $L b$. rhamnosus GG during storage of fermented cereal and pseudocereal substrates.

\begin{tabular}{lccc}
\hline Substrate Gr $\left[\log \mathrm{cfu} \mathrm{g}^{-1} \mathrm{~h}^{-1}\right]$ & $N_{0}\left[\log \mathrm{cfug}^{-1}\right]$ & $N_{\max }\left[\log \mathrm{cfu} \mathrm{g}^{-1}\right]$ \\
\hline RF & $-0,00154^{\mathrm{d}}$ & $8,29^{\mathrm{e}}$ & $7,49^{\mathrm{d}}$ \\
RG & $0,00019^{\mathrm{i}}$ & $7,66^{\mathrm{b}}$ & $7,93^{\mathrm{g}}$ \\
BF & $-0,00300^{\mathrm{b}}$ & $8,59^{\mathrm{f}}$ & $7,15^{\mathrm{c}}$ \\
WBF & $0,00024^{\mathrm{j}}$ & $8,06^{\mathrm{d}}$ & $7,69^{\mathrm{e}}$ \\
AF & $-0,00188^{\mathrm{c}}$ & $7,96^{\mathrm{d}}$ & $6,70^{\mathrm{b}}$ \\
AG & $-0,00031^{\mathrm{h}}$ & $8,02^{\mathrm{d}}$ & $7,79^{\mathrm{f}}$ \\
BWF & $-0,00076^{\mathrm{e}}$ & $7,91^{\mathrm{d}}$ & $7,46^{\mathrm{d}}$ \\
WBWF & $-0,00517^{\mathrm{a}}$ & $7,80^{\mathrm{c}}$ & $5,85^{\mathrm{a}}$ \\
WOF & $-0,00037^{\mathrm{g}}$ & $7,35^{\mathrm{a}}$ & $7,41^{\mathrm{d}}$ \\
MG & $-0,00048^{\mathrm{f}}$ & $7,83^{\mathrm{c}}$ & $7,60^{\mathrm{e}}$ \\
\hline
\end{tabular}

RF: rye flour, RG: rye grain, BF: barley flour, WBF: whole barley flour, $\mathrm{AF}$ : amaranth flour, AG: amaranth grain, BWF: buckwheat flour, WBWF: whole buckwheat flour, WOF: whole oat flour, MG: millet grain.

Gr: growth rate, $N_{0}$ : initial density of $L b$. rhamnosus GG, $N_{\max }$ : final density of Lb. rhamnosus GG.

${ }^{a-j}$ Means within a column with different superscript letters are significantly different $(P<0.05)$.

TABLE 7: Parameters of $\mathrm{pH}$ changes during storage of fermented cereal and pseudocereal substrates.

\begin{tabular}{lccc}
\hline Substrate & $k_{\mathrm{pH}}\left[\mathrm{h}^{-1}\right]$ & $\mathrm{pH}_{0}$ & $\mathrm{pH}_{\text {end }}$ \\
\hline RF & $0,00033^{\mathrm{g}}$ & $4,89^{\mathrm{b}}$ & $5,22^{\mathrm{c}}$ \\
RG & $0,00018^{\mathrm{f}}$ & $5,34^{\mathrm{d}}$ & $5,47^{\mathrm{d}}$ \\
BF & $0,00005^{\mathrm{e}}$ & $4,78^{\mathrm{b}}$ & $4,82^{\mathrm{b}}$ \\
WBF & $-0,00061^{\mathrm{b}}$ & $5,38^{\mathrm{d}}$ & $5,13^{\mathrm{c}}$ \\
AF & $-0,00435^{\mathrm{a}}$ & $6,17^{\mathrm{e}}$ & $4,52^{\mathrm{a}}$ \\
AG & $-0,00077^{\mathrm{b}}$ & $5,48^{\mathrm{d}}$ & $5,39^{\mathrm{c}}$ \\
BWF & $-0,00016^{\mathrm{c}}$ & $4,58^{\mathrm{a}}$ & $4,49^{\mathrm{a}}$ \\
WBWF & $-0,00677^{\mathrm{a}}$ & $5,50^{\mathrm{d}}$ & $4,87^{\mathrm{b}}$ \\
WOF & $-0,00004^{\mathrm{d}}$ & $5,12^{\mathrm{c}}$ & $5,15^{\mathrm{c}}$ \\
MG & $0,00022^{\mathrm{f}}$ & $5,23^{\mathrm{c}}$ & $5,30^{\mathrm{c}}$ \\
\hline
\end{tabular}

RF: rye flour, RG: rye grain, BF: barley flour, WBF: whole barley flour, AF: amaranth flour, AG: amaranth grain, BWF: buckwheat flour, WBWF: whole buckwheat flour, WOF: whole oat flour, MG: millet grain.

$k_{\mathrm{pH}}$ : rate of changes in $\mathrm{pH}$ values, $\mathrm{pH}_{0}$ : initial $\mathrm{pH}, \mathrm{pH}_{\text {end }}$ : final $\mathrm{pH}$.

${ }^{\mathrm{a}-\mathrm{g}}$ Means within a column with different superscript letters are significantly different $(P<0.05)$.

improve sensory quality of end products. We also found out that fermented cereal and pseudocereal substrates were stable during the 21-day storage period, except those prepared from amaranth flour, due to its rich nutritional composition. We are able to summarize that selected cereals and pseudocereals are suitable for developing new probiotic foods, which is in our interest.

\section{Conflict of Interests}

The authors declare that they have no conflict of interests.
TABLE 8: Parameters of lactic acid concentration changes during storage of fermented cereal and pseudocereal substrates.

\begin{tabular}{lccc}
\hline Substrate & $k_{\text {acid }}\left[\mathrm{mg} \mathrm{kg}^{-1} \mathrm{~h}^{-1}\right]$ & $c_{0}\left[\mathrm{mg} \mathrm{kg}^{-1}\right]$ & $c_{\text {end }}\left[\mathrm{mg} \mathrm{kg}^{-1}\right]$ \\
\hline RF & $-0,041^{\mathrm{c}}$ & $276,52^{\mathrm{a}}$ & $236,41^{\mathrm{a}}$ \\
RG & $0,126^{\mathrm{g}}$ & $404,90^{\mathrm{c}}$ & $401,05^{\mathrm{d}}$ \\
BF & $-0,122^{\mathrm{a}}$ & $469,20^{\mathrm{e}}$ & $361,41^{\mathrm{c}}$ \\
WBF & $-0,060^{\mathrm{b}}$ & $617,95^{\mathrm{f}}$ & $603,81^{\mathrm{f}}$ \\
AF & $3,752^{\mathrm{i}}$ & $402,90^{\mathrm{c}}$ & $2217,50^{\mathrm{i}}$ \\
AG & $0,092^{\mathrm{f}}$ & $883,81^{\mathrm{g}}$ & $925,48^{\mathrm{g}}$ \\
BWF & $-0,011^{\mathrm{d}}$ & $395,75^{\mathrm{c}}$ & $415,53^{\mathrm{d}}$ \\
WBWF & $4,967^{\mathrm{j}}$ & $416,12^{\mathrm{d}}$ & $1122,91^{\mathrm{h}}$ \\
WOF & $0,079^{\mathrm{e}}$ & $255,74^{\mathrm{a}}$ & $279,09^{\mathrm{b}}$ \\
MG & $2,455^{\mathrm{h}}$ & $359,89^{\mathrm{b}}$ & $483,38^{\mathrm{e}}$ \\
\hline
\end{tabular}

RF: rye flour, RG: rye grain, $\mathrm{BF}$ : barley flour, WBF: whole barley flour, AF: amaranth flour, AG: amaranth grain, BWF: buckwheat flour, WBWF: whole buckwheat flour, WOF: whole oat flour, MG: millet grain.

$k_{\text {acid }}$ : rate of lactic acid concentration changes, $c_{0}$ : initial concentration of lactic acid, $c_{\text {end }}$ : final concentration of lactic acid.

${ }^{\mathrm{a}-\mathrm{i}}$ Means within a column with different superscript letters are significantly different $(P<0.05)$.

TABLE 9: Parameters of acetic acid concentration changes during storage of fermented cereal and pseudocereal substrates.

\begin{tabular}{lccc}
\hline Substrate & $k_{\text {acid }}\left[\mathrm{mg} \mathrm{kg}^{-1} \mathrm{~h}^{-1}\right]$ & $c_{0}\left[\mathrm{mg} \mathrm{kg}^{-1}\right]$ & $c_{\text {end }}\left[\mathrm{mg} \mathrm{kg}^{-1}\right]$ \\
\hline RF & $0,016^{\mathrm{d}}$ & $60,60^{\mathrm{b}}$ & $63,15^{\mathrm{b}}$ \\
RG & $0,069^{\mathrm{f}}$ & $94,91^{\mathrm{d}}$ & $117,07^{\mathrm{e}}$ \\
BF & $0,007^{\mathrm{c}}$ & $88,67^{\mathrm{d}}$ & $78,48^{\mathrm{c}}$ \\
WBF & $0,058^{\mathrm{e}}$ & $94,44^{\mathrm{d}}$ & $120,77^{\mathrm{e}}$ \\
AF & $-0,091^{\mathrm{b}}$ & $258,30^{\mathrm{f}}$ & $227,02^{\mathrm{f}}$ \\
AG & $0,098^{\mathrm{g}}$ & $162,46^{\mathrm{e}}$ & $225,97^{\mathrm{f}}$ \\
BWF & $6,793^{\mathrm{h}}$ & $0,05^{\mathrm{a}}$ & $40,87^{\mathrm{a}}$ \\
WBWF & $-0,236^{\mathrm{a}}$ & $60,94^{\mathrm{b}}$ & $37,78^{\mathrm{a}}$ \\
WOF & $0,052^{\mathrm{e}}$ & $92,55^{\mathrm{d}}$ & $117,28^{\mathrm{e}}$ \\
MG & $0,015^{\mathrm{d}}$ & $82,33^{\mathrm{c}}$ & $90,11^{\mathrm{d}}$ \\
\hline
\end{tabular}

RF: rye flour, RG: rye grain, BF: barley flour, WBF: whole barley flour, AF: amaranth flour, AG: amaranth grain, BWF: buckwheat flour, WBWF: whole buckwheat flour, WOF: whole oat flour, MG: millet grain.

$k_{\text {acid }}$ : rate of acetic acid concentration changes, $c_{0}$ : initial concentration of acetic acid, $c_{\text {end }}$ : final concentration of acetic acid.

${ }^{\mathrm{a}-\mathrm{h}}$ Means within a column with different superscript letters are significantly different $(P<0.05)$.

\section{Acknowledgments}

The work was supported by The Scientific Grant Agency in Project-The Mutual Relations among Undesirable and Health Promoting Microorganisms in Cereal and Milk Matrices Fermented by Lactic Acid Bacteria: The Quantitative Analysis Leading to the Development of Fermented Products for people with Nutritional Handicaps (VEGA 1/0495/13). Financial support for the project was from the Slovak University of Technology Programme for the young researchers support: Potential of Cereals, Pseudocereals and Leguminous Substrates for Growth and Metabolic Activity of Probiotic Strains (ZAK 6425). 
TABLE 10: Parameters of citric acid concentration changes during storage of fermented cereal and pseudocereal substrates.

\begin{tabular}{lccc}
\hline Substrate & $k_{\text {acid }}\left[\mathrm{mg} \mathrm{kg}^{-1} \mathrm{~h}^{-1}\right]$ & $c_{0}\left[\mathrm{mg} \mathrm{kg}^{-1}\right]$ & $c_{\text {end }}\left[\mathrm{mg} \mathrm{kg}^{-1}\right]$ \\
\hline RF & $-0,014^{\mathrm{d}}$ & $170,02^{\mathrm{b}}$ & $155,95^{\mathrm{a}}$ \\
RG & - & - & - \\
BF & $-0,066^{\mathrm{f}}$ & $216,76^{\mathrm{c}}$ & $165,06^{\mathrm{a}}$ \\
WBF & $-0,028^{\mathrm{e}}$ & $478,37^{\mathrm{d}}$ & $470,37^{\mathrm{d}}$ \\
AF & $-0,732^{\mathrm{g}}$ & $1121,94^{\mathrm{f}}$ & $822,96^{\mathrm{f}}$ \\
AG & $0,120^{\mathrm{b}}$ & $515,66^{\mathrm{d}}$ & $581,54^{\mathrm{e}}$ \\
BWF & $0,003^{\mathrm{a}}$ & $130,93^{\mathrm{a}}$ & $136,86^{\mathrm{a}}$ \\
WBWF & $-3,161^{\mathrm{h}}$ & $557,95^{\mathrm{e}}$ & $429,94^{\mathrm{c}}$ \\
WOF & - & - & - \\
MG & $0,037^{\mathrm{c}}$ & $228,06^{\mathrm{c}}$ & $249,15^{\mathrm{b}}$ \\
\hline
\end{tabular}

RF: rye flour, RG: rye grain, BF: barley flour, WBF: whole barley flour, AF: amaranth flour, AG: amaranth grain, BWF: buckwheat flour, WBWF: whole buckwheat flour, WOF: whole oat flour, MG: millet grain.

$k_{\text {acid }}$ : rate of citric acid concentration changes, $c_{0}$ : initial concentration of citric acid, $c_{\text {end }}$ : final concentration of citric acid.

${ }^{\mathrm{a}-\mathrm{h}}$ Means within a column with different superscript letters are significantly different $(P<0.05)$.

\section{References}

[1] C. Wrigley, "Cereals/overview," in Encyclopedia of Grain Science, C. Wrigley, Ed., pp. 187-201, Elsevier Academic Press, Oxford, UK, 2004.

[2] R. J. Fletcher, "Pseudocereals/overview," in Encyclopedia of Grain Science, C. Wrigley, Ed., pp. 488-493, Elsevier Academic Press, Oxford, UK, 2004.

[3] A. Corsetti and L. Settanni, "Lactobacilli in sourdough fermentation," Food Research International, vol. 40, no. 5, pp. 539-558, 2007.

[4] A. Blandino, M. E. Al-Aseeri, S. S. Pandiella, D. Cantero, and C. Webb, "Cereal-based fermented foods and beverages," Food Research International, vol. 36, no. 6, pp. 527-543, 2003.

[5] B. G. Swanson, "Tannins and polyphenols," in Encyclopedia of Food Science and Nutrition, B. Cabalero, P. Finglas, and L. Trugo, Eds., pp. 5729-5733, Academic Press, Oxford, UK, 2003.

[6] R. W. Hutkins, Microbiology and Technology of Fermented Foods, Blackwell Publishing, Oxford, UK, 2006.

[7] J. A. Navrhus and T. Sørhaug, in Bakery and Cereal Products, Food Chemistry and Food Processing, Blackwell Publishing, Oxford, UK, 2006.

[8] W. Messens and L. De Vuyst, "Inhibitory substances produced by Lactobacilli isolated from sourdoughs-a review," International Journal of Food Microbiology, vol. 72, no. 1-2, pp. 31-43, 2002.

[9] S. Salminen A, "Probiotics: safety and efficacy," in Lactic Acid Bacteria. Microbiological and Functional Aspects, S. Lathinen, A. C. Ouwehand, S. Salminen, and A. Von Wright, Eds., pp. 689704, CRC Press, Boca Raton, Fla, USA, 2011.

[10] Ľ. Valík, A. Medvedová, and D. Liptáková, "Characterization of the growth of Lactobacillus rhamnosus GG in milk at suboptimal temperatures," Journal of Food and Nutrition Research, vol. 47, no. 1, pp. 60-67, 2008.

[11] B. D. Jyoti, A. K. Suresh, and K. V. Venkatesh, "Diacetyl production and growth of Lactobaciilus rhamnosus on multiple substrates," World Journal of Microbiology and Biotechnology, vol. 19, no. 5, pp. 509-514, 2003.
[12] E. K. Y. Lam, E. K. K. Tai, M. W. L. Koo et al., "Enhancement of gastric mucosal integrity by Lactobacillus rhamnosus GG," Life Sciences, vol. 80, no. 23, pp. 2128-2136, 2007.

[13] T. Mattila-Sandholm, S. Blum, J. K. Collins et al., "Probiotics: towards demonstrating efficacy," Trends in Food Science and Technology, vol. 10, no. 12, pp. 393-399, 1999.

[14] M. C. Collado, J. Meriluoto, and S. Salminen, "In vitro analysis of probiotic strain combinations to inhibit pathogen adhesion to human intestinal mucus," Food Research International, vol. 40, no. 5, pp. 629-636, 2007.

[15] EFSA Scientific Panel on Dietetic Products, Nutrition and Allergies Unit, "Consolidated list of Article 13 health claims. List of references received by EFSA. Parma," 2013, http://www.efsa .europa.eu/en/topics/topic/article13.htm.

[16] M. Kocková, J. Mendel, A. Medveďová, E. Šturdík, and Ľ. Valík, "Cereals and pseudocereals as a substrates for growth and metabolism of a probiotic strain Lactobacillus rhamnosus GG," Journal of Food and Nutrition Research, vol. 52, no. 1, pp. 25-36, 2013.

[17] J. Baranyi and T. A. Roberts, "A dynamic approach to predicting bacterial growth in food," International Journal of Food Microbiology, vol. 23, no. 3-4, pp. 277-294, 1994.

[18] M. H. Helland, T. Wicklund, and J. A. Narvhus, "Growth and metabolism of selected strains of probiotic bacteria in milk- and water-based cereal puddings," International Dairy Journal, vol. 14, no. 11, pp. 957-965, 2004.

[19] M. H. Helland, T. Wicklund, and J. A. Narvhus, "Growth and metabolism of selected strains of probiotic bacteria, in maize porridge with added malted barley," International Journal of Food Microbiology, vol. 91, no. 3, pp. 305-313, 2004.

[20] J. Pelikánová, D. Liptáková, Ľ. Valík, and K. Stančeková, "Evaluation of the growth of selected Lactobacilli in pseudocereal substrate," Potravinarstvo, vol. 5, no. 4, pp. 53-57, 2011.

[21] Z. Zalán, J. Hudáček, J. Štětina, J. Chumchalová, and A. Halász, "Production of organic acids by Lactobacillus strains in three different media," European Food Research and Technology, vol. 230, no. 3, pp. 395-404, 2010.

[22] A. Angelov, V. Gotcheva, R. Kuncheva, and T. Hristozova, "Development of a new oat-based probiotic drink," International Journal of Food Microbiology, vol. 112, no. 1, pp. 75-80, 2006. 

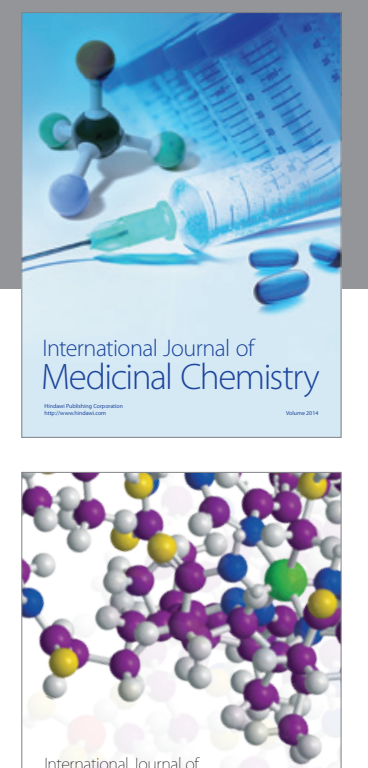

\section{Carbohydrate} Chemistry

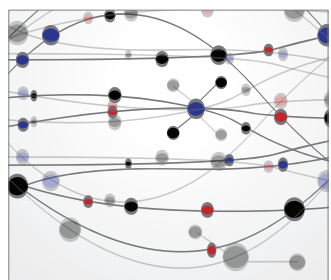

The Scientific World Journal
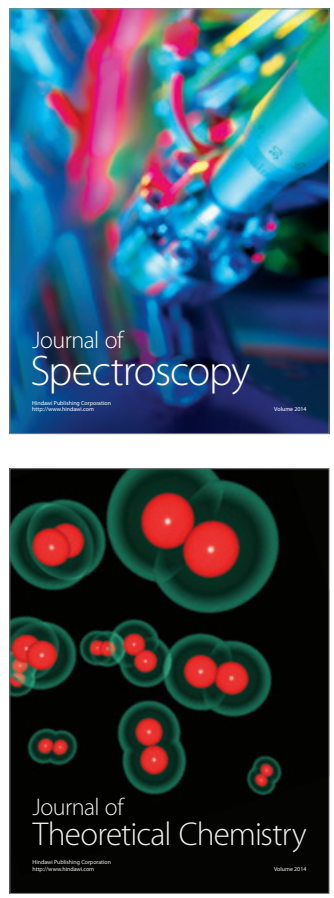
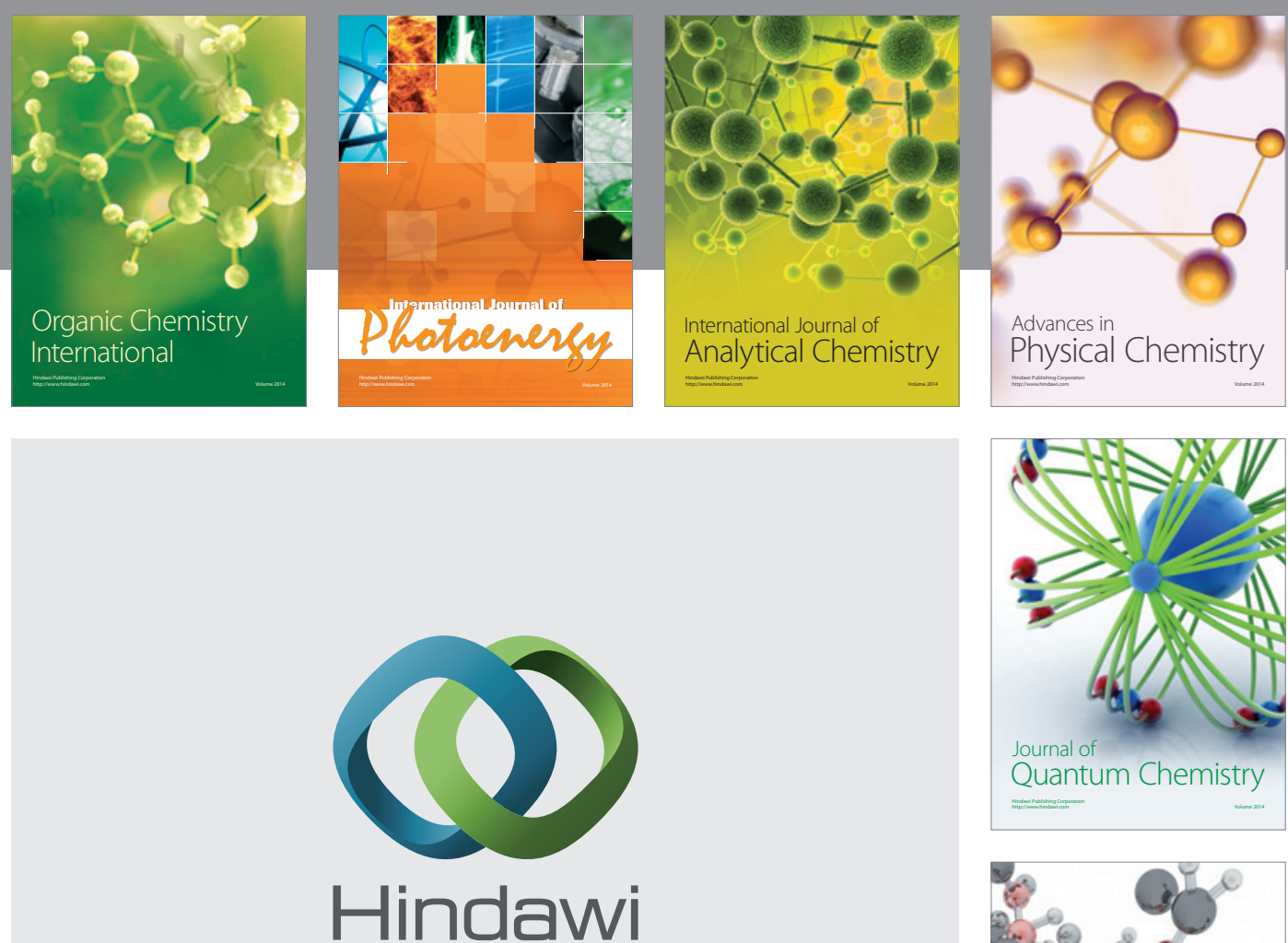

Submit your manuscripts at

http://www.hindawi.com

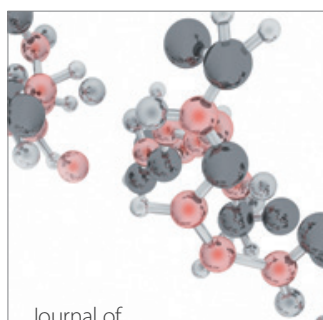

Analytical Methods

in Chemistry

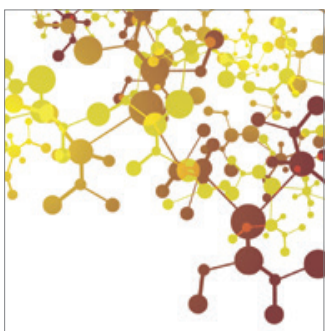

Journal of

Applied Chemistry

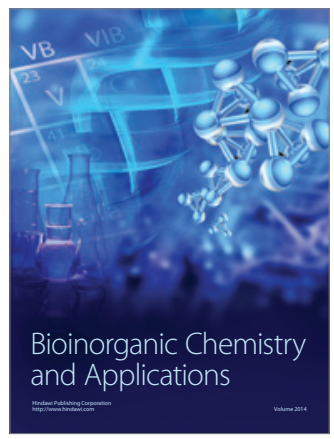

Inorganic Chemistry
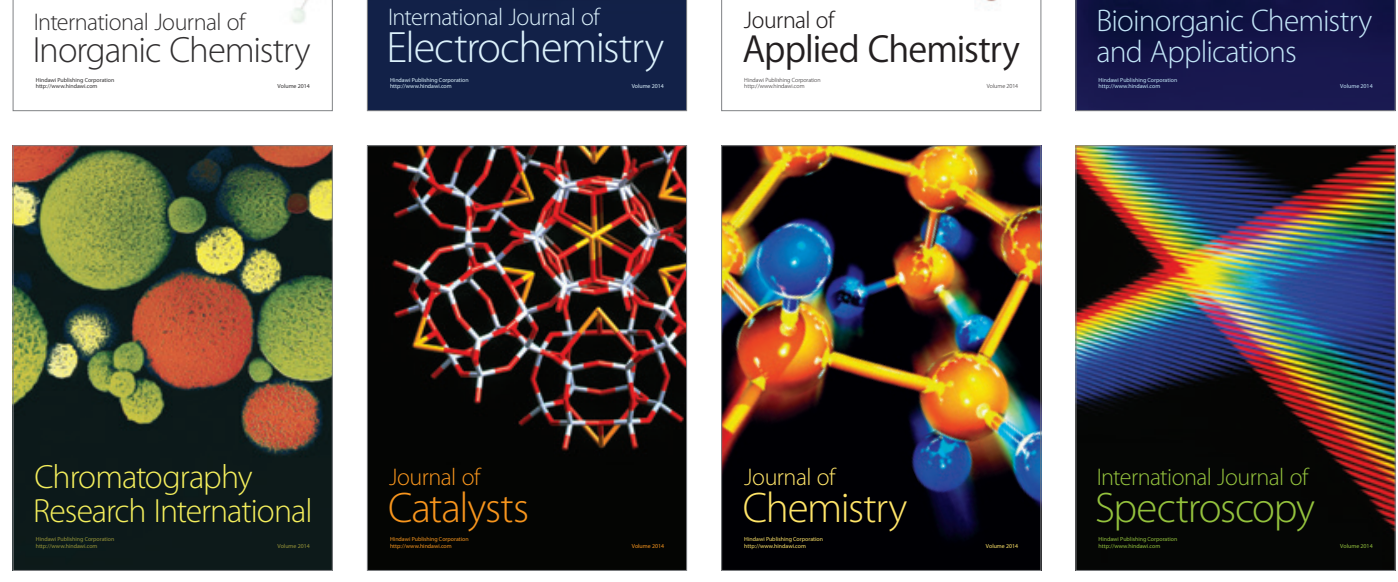